\title{
Carcinoma de seno y cardiotoxicidad
}

\author{
Carcinoma of the breast and cardiotoxicity \\ Heidy Roncancio-Martínez ${ }^{1}$ y Mónica Jaramillo-Jaramillo2* \\ ${ }^{1}$ Departamento de Cardiología Clínica, Clínica del Country; ${ }^{2}$ Facultad de Medicina, Universidad de los Andes. Bogotá, Colombia
}

\section{Resumen}

En las mujeres, la enfermedad cardiovascular y el cáncer de mama representan respectivamente la primera y la quinta causas de mortalidad, y tienen varios factores en común, como la edad, la dieta, la obesidad, el tabaquismo y el sedentarismo. En el cáncer de mama, el diagnóstico temprano y el tratamiento multimodal han mejorado el pronóstico y la supervivencia a largo plazo. Sin embargo, el cáncer de mama y las terapias utilizadas para su control (quimioterapia y radioterapia) pueden tener un impacto negativo en el sistema cardiovascular y producir alteraciones como disfunción ventricular, arritmias, miocarditis o enfermedad cardiovascular aterosclerótica acelerada. Cobran vital importancia la prevención, el diagnóstico y el tratamiento de la disfunción cardiaca y de otras complicaciones cardiovasculares, ya que para algunas la mortalidad es mayor que la del cáncer de mama. Es fundamental que toda paciente con cáncer de mama que vaya a iniciar tratamiento por oncología sea evaluada en cuanto a sus factores de riesgo para enfermedad cardiovascular. Los marcadores séricos, como la troponina y el péptido natriurético $B$, junto con el electrocardiograma y el ecocardiograma, pueden ser utilizados para la monitorización y la prevención de la cardiotoxicidad. Se resumen las recomendaciones para el diagnóstico y el tratamiento de la enfermedad cardiovascular de las pacientes en tratamiento para cáncer de mama.

Palabras clave: Cardiotoxicidad. Insuficiencia cardiaca. Carcinoma de mama. Enfermedad cardiovascular.

\begin{abstract}
Cardiovascular disease and breast cancer are the first and fifth cause of death among women worldwide, respectively. These diseases share several risk factors (age, diet, obesity, smoking, etc.) and early diagnosis and treatment have led to improving prognosis and survival. However, the increase in the risk of developing cardiotoxicity (manifested as ventricular dysfunction, arrhythmias, cardiovascular disease, etc.) associated with breast cancer treatment (chemotherapy and radiotherapy) could limit its use. Accordingly, there is an increasing importance of optimizing the prevention, diagnosis and treatment of cardiac dysfunction and cardiovascular complications developed as a result of breast cancer treatment. Risk factors for cardiovascular disease should be assessed for every patient with breast cancer scheduled to receive oncology treatment. This evaluation could include an electrocardiogram, an echocardiogram and biomarkers such as troponin levels and BNP in order to perform a risk assessment and monitoring of cardiotoxicity. Here, we summarize the recommendations regarding the diagnosis and management of breast cancer patients at risk of developing treatment-induced cardiotoxicity.
\end{abstract}

Key words: Cardiotoxicity. Heart failure. Breast carcinoma. Cardiovascular disease.

\section{Correspondencia:}

*Monica Jaramillo-Jaramillo

E-mail: mjaramil@uniandes.edu.co
Fecha de recepción: 10-05-2020

Fecha de aceptación: 11-03-2021

DOI: 10.24875/RCCAR.M21000048
Disponible en internet: 29-07-2021 Rev Colomb Cardiol. 2021;28(3):207-216 www.rccardiologia.com 0120-5633 / C 2020 Sociedad Colombiana de Cardiología y Cirugía Cardiovascular. Publicado por Permanyer. Este es un artículo open access bajo la licencia CC BY-NC-ND (http://creativecommons.org/licenses/by-nc-nd/4.0/). 


\section{Introducción y consideraciones epidemiológicas}

La enfermedad cardiovascular (ECV) es la principal causa de muerte en las mujeres y comparte con el cáncer de mama (CM) varios factores de riesgo (FR), como la edad, la dieta, la obesidad, el tabaquismo y la vida sedentaria, entre otras ${ }^{1}$.

En los Estados Unidos de América, la ECV afecta aproximadamente a 47.8 millones de mujeres y el CM aproximadamente a 3.32 millones ${ }^{1}$.

La incidencia de CM se ha incrementado un 3.1\% anualmente, desde 641,000 casos en 1980 hasta más de 1.6 millones de casos para $2010^{2}$.

En 2018, el CM fue la malignidad más diagnosticada y la principal causa de muerte relacionada con cáncer en las mujeres. Se estima que durante ese año se diagnosticaron cerca de 2.1 millones de nuevos casos de CM en los Estados Unidos de América, lo cual representa uno de cada cuatro cánceres diagnosticados ${ }^{3}$.

Los avances en el diagnóstico y el tratamiento del CM han llevado a un mejor pronóstico y una alta proporción de sobrevivientes a largo plazo. Casi el 90\% de las mujeres diagnosticadas de CM sobrevivirán más de 5 años ${ }^{4}$.

La incidencia varía entre los países de altos ingresos (92 por 100,000 habitantes en América del Norte) y los países de bajos ingresos (27 por 100,000 habitantes en África y Asia Oriental), debido a diferencias en los FR y a la disponibilidad de pruebas de detección temprana. En consecuencia, en los países de altos ingresos el CM suele ser diagnosticado en un estadio temprano y tiene mejor pronóstico que en los países de menores ingresos, lo cual se traduce en una mayor mortalidad en estos últimos ${ }^{2}$.

\section{Factores de riesgo para enfermedad cardiovascular y cáncer de mama}

El riesgo de desarrollar $\mathrm{CM}$ se incrementa con la edad, desde 1 de cada 49 mujeres entre el nacimiento y los 49 años hasta 1 de cada 14 mujeres si tienen 70 años o más ${ }^{5}$. Entre los FR establecidos para el desarrollo de $\mathrm{CM}$ se encuentran el antecedente familiar de la enfermedad, las mutaciones BRCA1 o 2, la nuliparidad, la menarquia temprana, la menopausia tardía, el consumo de alcohol, el sedentarismo, el sobrepeso, el uso de hormonas exógenas y la radiación en el tórax. La lactancia y la actividad física son factores protectores ${ }^{6}$.
Existen FR en común para el desarrollo de $\mathrm{CM}$ y enfermedad cardiovascular, que deben ser prevenidos y tratados.

Según la Organización Mundial de la Salud, en torno a un $30 \%$ de las muertes por cáncer se deben a cinco $\mathrm{FR}$, entre los que tienen gran importancia la alimentación (índice de masa corporal elevado, consumo insuficiente de frutas y verduras), el ejercicio físico (falta de ejercicio) y el consumo de tabaco y alcohol. El tabaquismo es el factor de riesgo que por sí solo provoca una mayor mortalidad por cáncer, aproximadamente un $22 \%$ de las muertes por cáncer en general (Fig. 1).

\section{Riesgo de cardiotoxicidad en pacientes con cáncer de mama}

El CM puede tener un impacto cardiovascular negativo (por ejemplo, disfunción ventricular y acelerar la ECV), y en las mujeres con ECV preexistente esto puede influenciar la toma de decisiones acerca del tratamiento del cáncer. Las mejoras en la detección y el tratamiento del CM han llevado a un aumento de la supervivencia de estas pacientes, pero también se ha visto que se ha incrementado el riesgo de complicaciones cardiacas a largo plazo secundarias a la quimioterapia.

Uno de los efectos secundarios más limitantes que generan la quimoterapia y la radioterapia es la cardiotoxicidad, y se conoce que el tratamiento oncológico y hematológico triplica el riesgo de eventos cardiovasculares a medio y largo plazo ${ }^{7}$. Limitar el tratamiento oncológico debido a la aparición de toxicidad cardiovascular puede impedir usar terapias potencialmente curativas, mientras que infraestimar este riesgo puede comprometer la supervivencia a largo plazo ${ }^{8}$.

Para las mujeres mayores, la ECV representa una mayor amenaza de mortalidad que el $\mathrm{CM}^{1}$.

Varias terapias adyuvantes contra el CM pueden causar una amplia gama de complicaciones cardiacas agudas y tardías; tema actual de preocupación teniendo en cuenta el aumento del número de sobrevivientes de CM a largo plazo y la tendencia a combinar dos o más terapias potencialmente cardiotóxicas en el contexto adyuvante ${ }^{4}$.

El pronóstico de las mujeres mayores con $\mathrm{CM}$ se ve afectado por el manejo efectivo de su comorbilidad, que incluye hipertensión arterial y diabetes mellitus. Si no se identifican ni se tratan los FR cardiovasculares se puede generar un riesgo mayor para la salud que el CM por sí solo. 


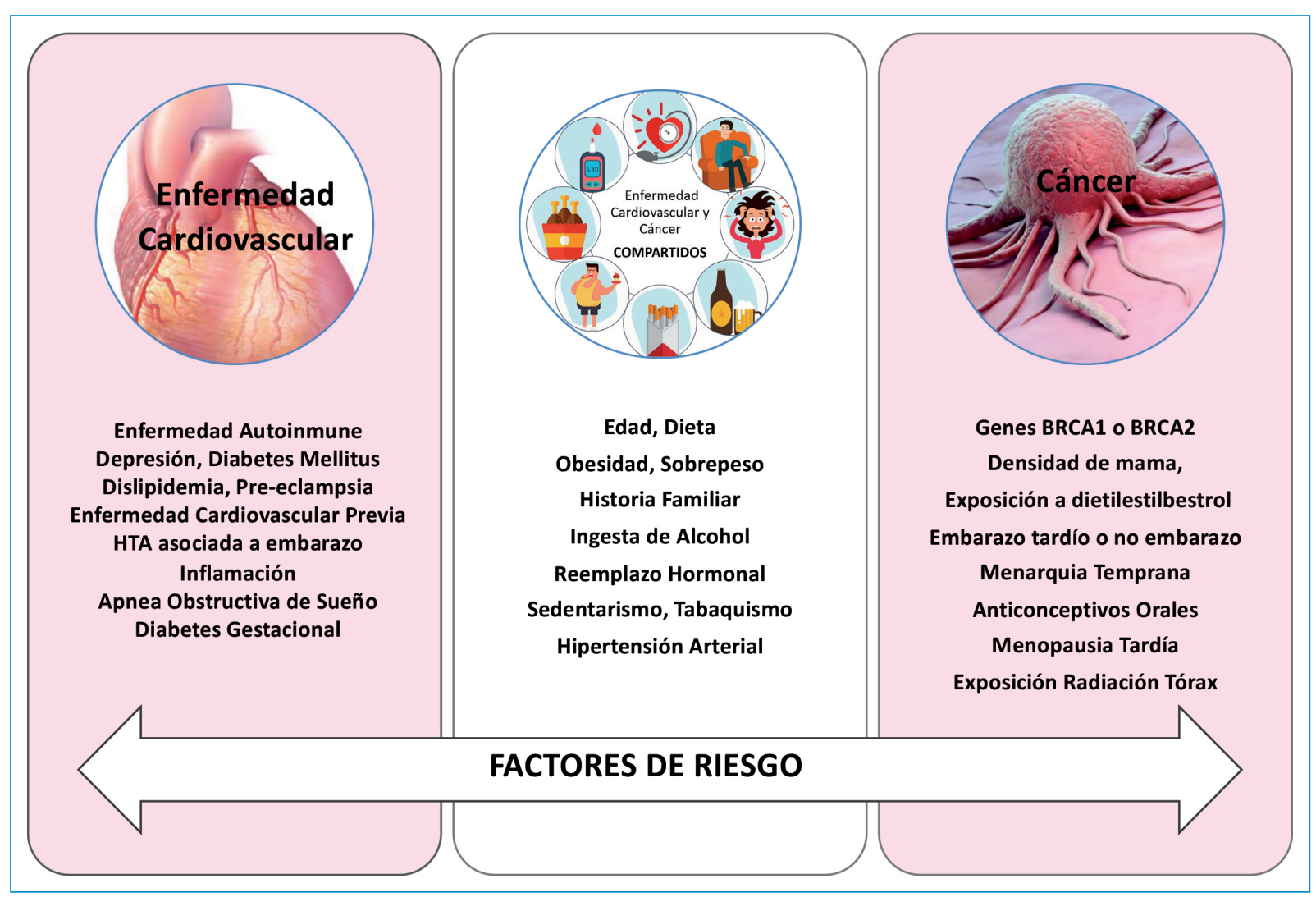

Figura 1. Intersección entre factores de riesgo para enfermedad cardiovascular y cáncer de mama. Contenido adaptado de: Mehta LS, et al. ${ }^{1}$

\section{Efectos cardiovasculares del tratamiento del cáncer de mama}

El tratamiento del CM puede dar lugar a distintos efectos secundarios cardiovasculares, que pueden ser agudos o tardíos, reversibles o irreversibles. Se han descrito desde disfunción del ventrículo izquierdo e insuficiencia cardiaca hasta arritmias e isquemia miocárdica'.

Cada tratamiento empleado tiene distintos efectos cardiovasculares (Tabla 1). El riesgo de cardiotoxicidad inducida por antraciclinas depende de la dosis acumulativa. Por ejemplo, la doxorubicina se asocia con una incidencia de falla cardiaca ${ }^{9}$ del $3 \%$ al $5 \%$ con una dosis acumulada de $400 \mathrm{mg} / \mathrm{m}^{2}$, del $7 \%$ al $26 \%$ con dosis de $550 \mathrm{mg} / \mathrm{m}^{2}$, y del $18 \%$ al $48 \%$ con dosis de $700 \mathrm{mg} / \mathrm{m}^{2}$.

En un metaanálisis que incluyó una cohorte de unos 29,000 pacientes se observó cardiotoxicidad grave asociada con trastuzumab en aproximadamente el $3 \%$ de ellos, con una incidencia creciente hasta el 19\% entre pacientes mayores, fumadores y con diabetes, hipertensión o ECV ${ }^{9,10}$.

\section{Monitorización y prevención de la cardiotoxicidad}

Las mujeres con $\mathrm{CM}$ y aquellas con ECV comparten numerosos $\mathrm{FR}^{1,4}$. Actualmente no hay escalas prospectivas que valoren de forma conjunta el riesgo cardiovascular y de cardiotoxicidad, y las escalas tradicionales subestiman el riesgo asociado al tratamiento del cán$\operatorname{cer}^{11}$. Sin embargo, se recomienda estratificar el riesgo cardiovascular usando las escalas disponibles antes de iniciar el tratamiento antitumoral, y evaluar la presencia de FR que aumentan el riesgo de eventos cardiovasculares durante el tratamiento antitumoral ${ }^{12}$.

El tratamiento de los FR cardiovasculares en cualquier paciente es importante y ello es igualmente válido para la población de pacientes con cáncer ${ }^{13}$. El esquema de monitorización varía según los esquemas del tratamiento del cáncer.

\section{Biomarcadores}

En situaciones seleccionadas, los biomarcadores cardiacos pueden ayudar a detectar 0 predecir 
Tabla 1. Tratamiento del cáncer y efectos adversos cardiovasculares

\begin{tabular}{|c|c|}
\hline $\begin{array}{l}\text { Agentes } \\
\text { quimioterapéuticos. }\end{array}$ & Efecto cardiovascular \\
\hline $\begin{array}{l}\text { Antracíclicos } \\
\text { (eg, doxorrubicina, } \\
\text { epirrubicina). }\end{array}$ & $\begin{array}{l}\text { Disfunción ventricular izquierda, falla } \\
\text { cardiaca, miocarditis, pericarditis, } \\
\text { fibrilación auricular, taquicardia } \\
\text { ventricular, fibrilación ventricular }\end{array}$ \\
\hline $\begin{array}{l}\text { Agentes alquilantes: } \\
\text { (p. ej., cisplatino, } \\
\text { ciclofosfamida) }\end{array}$ & $\begin{array}{l}\text { Disfunción ventricular izquierda, falla } \\
\text { cardiaca, miocarditis, pericarditis, } \\
\text { trombosis arterial, bradicardia, } \\
\text { fibrilación auricular, taquicardia } \\
\text { supraventricular. }\end{array}$ \\
\hline $\begin{array}{l}\text { Taxanos: } \\
\text { (p. ej., paclitaxel) }\end{array}$ & $\begin{array}{l}\text { Bradicardia, bloqueo cardíaco, } \\
\text { extrasístoles ventriculares. }\end{array}$ \\
\hline $\begin{array}{l}\text { Antimetabolitos } \\
\text { (p. ej., 5-fluorouracilo, } \\
\text { capecitabina) }\end{array}$ & $\begin{array}{l}\text { Trombosis coronaria, } \\
\text { Espasmo coronario, fibrilación } \\
\text { auricular, taquicardia y fibrilación } \\
\text { ventricular. }\end{array}$ \\
\hline $\begin{array}{l}\text { Terapia } \\
\text { neurohormonal. } \\
\text { (p. ej., tamoxifeno, } \\
\text { anastrozol, } \\
\text { letrozol) }\end{array}$ & $\begin{array}{l}\text { Trombosis venosa, tromboembolismo, } \\
\text { Enfermedad arterial periférica, arritmia, } \\
\text { Enfermedad valvular, pericarditis, } \\
\text { falla cardiaca. }\end{array}$ \\
\hline $\begin{array}{l}\text { Terapias dirigidas por } \\
\text { HER-2 } \\
\text { (p. ej., trastuzumab, } \\
\text { pertuzumab) }\end{array}$ & Disfunción ventricular izquierda. \\
\hline $\begin{array}{l}\text { Quinasa dependiente } \\
\text { de ciclina } 4 / 6 \\
\text { Inhibidores (p. ej., } \\
\text { palbociclib, ribociclib) }\end{array}$ & Prolongación QTc. \\
\hline Radioterapia & $\begin{array}{l}\text { Enfermedad coronaria. miocarditis, } \\
\text { enfermedad valvular, pericarditis. }\end{array}$ \\
\hline
\end{tabular}

toxicidades cardiovasculares, en particular cardiomiopatía e insuficiencia cardiaca. El papel exacto y el momento de la medición de los biomarcadores en cada paciente sometida a quimioterapia con medicamentos potencialmente cardiotóxicos aún no se ha determinado ${ }^{14}$.

La troponina y el péptido natriurético $B$ están siendo utilizados de manera no sistemática para la identificación temprana, la estratificación del riesgo y la monitorización de la cardiotoxicidad relacionada con la quimioterapia. En el tratamiento del $\mathrm{CM}$, la troponina es un marcador sensible y específico de lesión miocárdica en los pacientes tratados con quimioterapia, y lo mismo ocurre con el péptido natriurético $B$. Varios estudios concluyen que el incremento de las cifras de troponina y de péptido natriurético $B$ indica lesión subclínica $^{15}$.
El momento específico para medir los biomarcadores cardiacos en relación con la troponina varía significativamente en diferentes estudios. En pacientes seleccionados de alto riesgo, o en aquellos que reciben altas dosis de quimioterapia potencialmente cardiotóxica (en especial antraciclinas), debe considerarse la evaluación de los biomarcadores antes del inicio, ya que puede identificar a los pacientes con mayor riesgo para desarrollar disfunción cardiovascular ${ }^{14,16}$.

\section{Electrocardiograma basal}

La importancia de la prolongación del QTc como parámetro de seguridad de los medicamentos es ampliamente reconocida, y especial atención merecen los pacientes que recibirán inhibidores de la tirosina cinasa dependientes de la ciclina (ribociclib). Se debe obtener un electrocardiograma al inicio del tratamiento y realizar controles periódicos ${ }^{8}$.

\section{Ecocardiograma}

El ecocardiograma como método de monitorización para evaluar la función ventricular está indicado cuando se utilicen medicamentos con potencial de producir falla cardiaca o disfunción ventricular izquierda. En estos pacientes es obligatoria la evaluación basal de la fracción de eyección del ventrículo izquierdo (FEVI) y de la función diastólica; por ejemplo, en pacientes que reciben antraciclinas o trastuzumab ${ }^{8,14}$.

Identificar a los pacientes que tienen compromiso subclínico de la función ventricular es la estrategia ideal. Varios expertos recomiendan, para la detección de la disfunción subclínica del ventrículo izquierdo, realizar strain longitudinal global del ventrículo izquierdo, y si se observa un porcentaje relativo de reducción $>15 \%$ en el ecocardiograma de control con respecto al basal se considera anormal y predictor de falla cardiaca $^{17}$.

Para las pacientes con CM en tratamiento con terapia endocrina sin FR cardiovasculares no se recomienda el monitoreo sistemático de la toxicidad cardiovascular $^{8}$.

\section{Tratamiento de la enfermedad cardiovascular en las mujeres con cáncer de mama}

Entre las sobrevivientes de CM merece una atención especial la ECV, ya que representa la segunda causa de muerte en la población total y la primera en las 
mujeres posmenopáusicas con $\mathrm{CM}$ y receptores hormonales positivos $(\mathrm{RH}+)^{18}$. Además de los FR cardiovasculares preexistentes, algunas de las terapias usadas en el tratamiento del CM pueden afectar en forma directa o indirecta la función y la homeostasis cardiovasculares.

\section{Tratamiento multimodal contemporáneo en pacientes con cáncer de mama}

El tratamiento actual del CM incluye cirugía, radiación y tratamientos sistémicos que tienen como objetivo llegar a todas las células cancerosas del cuerpo, independientemente de su ubicación. Los tratamientos comprenden quimioterapia, terapia endocrina y terapia dirigida, con anticuerpos e inmunoterapia. La elección, el tiempo y la secuencia de tratamiento en cada paciente se basan en las características del tumor y los factores clínicos, incluyendo el estado funcional de la paciente y su comorbilidad ${ }^{8}$.

Las decisiones de tratamiento se basan en la biología del tumor y en factores clínicos. En términos generales, el CM puede dividirse en tres subgrupos diferentes: con receptores hormonales positivos $(\mathrm{HR}+)$, con receptores positivos del factor de crecimiento epidérmico humano (HER2 +) y triple negativo ${ }^{8}$.

El tratamiento multimodal se reserva para pacientes en estadio temprano en quienes la curación es la meta. La mayoría de las pacientes con enfermedad metastásica se consideran incurables y el objetivo es prolongar la vida manteniendo una buena calidad de esta.

La radiación disminuye el riesgo de mortalidad por $\mathrm{CM}$, pero puede aumentar la mortalidad no relacionada con el CM, debido a un exceso de muerte por ECV. El aumento del riesgo se observa tempranamente después de la exposición y continúa al menos durante 20 años ${ }^{19}$.

\section{Estrategias preventivas para minimizar el riesgo antes de iniciar el tratamiento}

- Evaluación global de los FR cardiovasculares y su manejo: el CM y la ECV comparten FR comunes. Se cree que aproximadamente el $80 \%$ de las ECV podrían prevenirse con la modificación de los FR, promoviendo una dieta saludable, actividad física, un peso saludable, abstinencia de tabaco, control de la presión arterial, manejo de la diabetes mellitus y un buen perfil lipídico. La adherencia a comportamientos de salud cardiovascular ideales de la campaña Life's Simple 7 Health de la American Heart
Association se asocia con una tendencia a la disminución del $\mathrm{CM}$, y el manejo intensivo de estos FR cardiovasculares puede reducir sustancialmente el riesgo de desarrollar cáncer. Modificar los FR cardiovasculares podría prevenir algunos casos de $\mathrm{CM}$ y disminuir la mortalidad de causa cardiovascular ${ }^{2}$. Se necesitan estrategias efectivas y viables para mitigar el riesgo de ECV en pacientes con cáncer. Se debe obtener la historia clínica completa, revisar las medicaciones actuales y la adherencia terapéutica, realizar una exploración física completa, desarrollar un plan individual de tratamiento para la paciente, verificar que esté tomando la medicación cardioprotectora apropiada, identificar los FR modificables, establecer metas para el control de los FR y un protocolo para evaluar su logro, desarrollar un plan que resuma las metas a largo plazo, e identificar el proveedor de salud responsable para el seguimiento de estas metas ${ }^{20}$.

Detección de los pacientes con riesgo aumentado de cardiotoxicidad, búsqueda de ECV y evaluación de la función cardiaca: el primer paso es una evaluación basal cuidadosa de los FR cardiovasculares mediante anamnesis, exploración física y medición basal de la función cardiaca. Los biomarcadores cardiacos (péptidos natriuréticos y troponinas) pueden ser considerados para compararlos durante el seguimiento. De acuerdo con el riesgo individual de las pacientes, en algunas se debe descartar enfermedad coronaria y en otras hay que realizar un electrocardiograma de 12 derivaciones para medición del QTC. Es crucial detectar anormalidades cardiacas subclínicas, las cuales pueden influir en las decisiones clínicas sobre la elección de la quimioterapia, la indicación de cardioprotección o la necesidad de un seguimiento más estricto. En caso de detectar ECV, esta debe tratarse. La evaluación del riesgo basal permite la interpretación apropiada de los resultados y de los cambios durante el monitoreo regular. Las pacientes con alto riesgo de presentar cardiotoxicidad deben ser examinadas por un cardiólogo experimentado, y si el riesgo de la paciente es muy elevado se evitará el uso de tratamientos cardiotóxicos ${ }^{21}$.

\section{Estrategias preventivas para minimizar el riesgo durante un tratamiento para el cáncer de mama potencialmente cardiotóxico}

- Estrategias oncológicas para mitigar la cardiotoxicidad: el acercamiento multidisciplinario para el 
cuidado de pacientes expuestos a tratamiento multimodalidad del cáncer ha resultado en un aumento del entendimiento del mecanismo de la cardiotoxicidad relacionada con el tratamiento. Entre las estrategias se encuentran:

- Dexrazoxano: es un agente quelante que liga el hierro intracelular, disminuyendo la formación de radicales libres y la apoptosis de los cardiomiocitos. Numerosos estudios en pacientes con $\mathrm{CM}$ usando doxorubicina o epirubicina mostraron reducciones significativas de compromiso de la FEVI o de presentación de insuficiencia cardiaca. Los estudios han informado una reducción de los eventos cardiacos del $65 \% 22$ y de la insuficiencia cardiaca del $79 \%$ al $82 \% \%^{19,23}$ cuando se incluye dexrazoxano en el tratamiento. Por la incertidumbre del riesgo de una segunda malignidad y la posibilidad de proporciones reducidas de respuesta del tumor, la indicación de la Food and Drug Administration para el dexrazoxano se limita a aquellas pacientes con dosis acumulativas de doxorubicina mayores de $300 \mathrm{mg} / \mathrm{m}^{2}$ y que continuarán recibiendo doxorubicina para el control del tumor. Las agencias europeas ${ }^{24,25}$ lo indican cuando se ha recibido un mínimo de epirubicina acumulada de $540 \mathrm{mg} / \mathrm{m}^{2}$.

- Administración de doxorubicina: en un metaanálisis se ha comparado la administración de doxorubicina en infusión $\geq 6$ horas frente a la administración en bolo, y se ha observado una reducción del $73 \%$ en los casos de insuficiencia cardiaca clínica ${ }^{26}$. La doxorubicina liposomal permite administrar una dosis acumulativa mayor con eficacia preservada y con menor frecuencia de efectos secundarios (odds ratio: 0.18; intervalo de confianza del 95\%: 0.08-0.38 ${ }^{27}$.

- Radiación: las técnicas de radiación modernas limitan la dosis de radicación total y la dosis por fracción, y disminuyen el volumen de corazón expuesto a la radiación. Estas técnicas probablemente se asocien con menores complicaciones ${ }^{1}$. Las pacientes con FR cardiovasculares tradicionales tienen un riesgo aumentado de ECV, por lo que controlar intensivamente estos FR mejora los resultados cardiovasculares en tales pacientes ${ }^{23}$.

- Tratamiento preventivo: el perfil de riesgo-beneficio de cada paciente debe tenerse en cuenta cuando se elige una terapia con probables efectos cardiotóxicos. No hay guías definitivas de prevención cardiovascular en pacientes con $\mathrm{CM}$, porque la mayoría de los estudios son pequeños y los objetivos primarios han sido variables.

- Betabloqueadores y bloqueadores del sistema renina-angiotensina-aldosterona: algunos estudios pequeños, aleatorizados y controlados con placebo han demostrado el beneficio de los betabloqueadores en pacientes con CM. Dos estudios ${ }^{28,29}$ observaron un menor compromiso de la FEVI a los 6 meses con terapia profiláctica con un betabloqueador (carvedilol o nebivolol) comparado con placebo antes del inicio de la quimioterapia basada en antraciclinas. Otro estudio ${ }^{30}$ encontró que las pacientes con $\mathrm{CM}$ y corazones estructuralmente normales al inicio del estudio que fueron tratadas con antraciclina o trastuzumab, y que estaban tomando betabloqueadores durante el tratamiento, tuvieron menor incidencia de nuevos eventos de insuficiencia cardiaca. Por el contrario, un estudio retrospectivo $^{31}$ de 179 pacientes que recibieron adyuvancia con trastuzumab para el CM temprano encontró que los betabloqueadores, los inhibidores de la enzima convertidora de angiotensina (IECA) y los bloqueadores del receptor de la angiotensina (ARB) no modificaban el riesgo de cardiotoxicidad inducida por trastuzumab. En balance, hay controversia sobre si se debe iniciar la profilaxis y con qué tipo de betabloqueadores antes del inicio del tratamiento con una antraciclina o con trastuzumab. El estudio PRA$\mathrm{DA}^{32}$ (de prevención de la disfunción cardiaca durante el tratamiento adyuvante del $\mathrm{CM}$ ) fue un ensayo aleatorizado, doble ciego, controlado con placebo, factorial $2 \times 2$ en pacientes con $\mathrm{CM}$ temprano que fueron sometidas a cirugía y quimioterapia adyuvante (5-fluorouracilo, epirubicina y ciclofosfamida). Las pacientes recibieron la combinación de candesartán, succinato de metoprolol y placebo. El succinato de metoprolol no mostró ningún efecto y en el grupo de candesartán hubo una reducción modesta de la declinación de la FEVI. EI estudio MANTICORE ${ }^{33}$ fue un estudio aleatorizado, doble ciego, controlado con placebo en pacientes con CM temprano que recibieron tratamiento adyuvante con trastuzumab; el $33 \%$ recibieron antraciclinas. Durante el tratamiento adyuvante, recibieron perindopril, bisoprolol o placebo (1:1:1). Hubo una pequeña declinación en la FEVI en el grupo de placebo (5\%), que se atenúo al $3 \%$ en el grupo de IECA y al $1 \%$ en el grupo de betabloqueador. Ninguno de los estudios evitó la remodelación cardia$\mathrm{ca}^{8}$. Por último, en un ensayo ${ }^{34}$ mayor con 468 pacientes con $\mathrm{CM}$ HER2+ que recibieron 
trastuzumab, de las cuales el $40 \%$ fueron tratadas simultáneamente con doxorubicina, se aleatorizaron 1:1:1 para recibir carvedilol, lisinopril o placebo. Las pacientes fueron evaluadas a los 2 años y se consideró que presentaban cardiotoxicidad si mostraban una disminución de la $\mathrm{FEVI}>10 \%$ o una disminución de la FEVI > 5\% con una FEVI absoluta $<50 \%$. Entre las pacientes con exposición previa a antraciclinas, el $31 \%$ de las que recibieron carvedilol, el $37 \%$ de las que recibieron lisinopril y el $47 \%$ de las que recibieron placebo experimentaron una declinación significativa de la FEVI. Tales resultados se consideraron estadísticamente significativos $(p=$ $0.009)^{28}$. Estos fueron estudios pequeños realizados en pacientes relativamente jóvenes, saludables y con poca comorbilidad, tienen diferentes definiciones de cardiotoxicidad, usaron medicaciones cardiacas diferentes y los seguimientos fueron cortos, lo cual limita su generalización. El beneficio clínico de prevenir pequeñas disminuciones de la FEVI en pacientes asintomáticas no está claro ${ }^{28}$. Durante el tratamiento, el inicio temprano del tratamiento para la cardiomiopatía inducida por antraciclinas, sin importar los síntomas, es benéfico. La detección temprana de la cardiotoxicidad inducida por antraciclinas y el tratamiento de la insuficiencia cardiaca se asocian a una mejoría de la FEVI. Los betabloqueadores también se han asociado con la recuperación de la función del ventrículo izquierdo en la cardiomiopatía inducida por trastuzumab ${ }^{8}$; se demostró una mejoría significativa de la FEVI, pero no el retorno a la basal. Para la cardiotoxicidad por trastuzumab, la Sociedad Europea de Cardiología y la Canadian Cardiovascular Society recomiendan el inicio de un IECA y un betabloqueador en pacientes con insuficiencia cardiaca y $\mathrm{FEVI}<40 \%$; también recomiendan iniciar un IECA en todos los pacientes asintomáticos con $\mathrm{FEVI}<40 \%$, y se deben considerar las terapias en pacientes asintomáticos en quienes la FEVI disminuya (> 10\% de disminución en comparación con el valor basal o $\mathrm{FEVI}<53 \%)^{8}$. Los estudios observacionales sugieren que el uso de betabloqueadores puede influir en la mortalidad $o$ en la recurrencia del $\mathrm{CM}$. Algunos estudios informan un aumento de la sobrevida y una disminución de recurrencia, otro halló un aumento de la recurrencia y uno obtuvo unos resultados neutros ${ }^{35}$. En estos estudios se hallan factores de confusión y se requiere un estudio aleatorizado controlado para evaluar el beneficio potencial de los betabloqueadores, los IECA y los ARB en sobrevivientes de CM. Los IECA son los agentes mejor estudiados para prevenir y tratar la disfunción ventricular izquierda, mientras que los ARB han sido menos estudiados en esta área. La espironolactona podría ser un agente protector lógico, y al menos un estudio ha demostrado atenuar la declinación en la FEVI y estabilizar la función diastólica ${ }^{36}$. Aunque no hay estudios aleatorizados grandes en pacientes con CM con betabloqueadores, IECA o ARB, parece razonable tratar a los pacientes con cardiomiopatía por antraciclina o trastuzumab de acuerdo con las guías para el manejo de la insuficiencia cardiaca $^{37}$.

- Sacubitril/valsartán: en un estudio aleatorizado prospectivo que comparó sacubitril/valsartán con candesartán en pacientes con CM y FEVI reducida previa a la administración de antraciclinas, el sacubitril/valsartán mostró un menor aumento del péptido natriurético $B$, una recuperación en la caminata de 6 minutos, una mayor supresión de las arritmias ventriculares y una mejoría de los indicadores de la función ventricular izquierda ${ }^{30}$.

- Estatinas: pueden tener propiedades pleiotrópicas y prevenir la cardiotoxicidad de las antraciclinas. Hay datos iniciales de una limitación de la disminución de la FEVI con dosis altas de estatinas ${ }^{30}$.

- Ejercicio: mitiga la cardiotoxicidad y los eventos adversos de la exposición a antraciclinas. El efecto cardioprotector del ejercicio aeróbico en la población general está bien establecido, pero el mecanismo específico de cardioprotección en el cáncer no se conoce por completo. El American College of Sports Medicine ha implementado unas guías de ejercicio. En la figura número 2 se resume lo expuesto anteriormente (Fig. 2) ${ }^{38,39}$.

- Terapia hormonal: es la piedra angular en el tratamiento del CM con receptores positivos para estrógenos. El tamoxifeno tiene un perfil de riesgo cardiaco mejor que los inhibidores de la aromatasa. Estos medicamentos alteran los lípidos, por lo que se recomienda realizar anualmente un tamizaje de lípidos y un monitoreo de la presión arterial. Las mujeres menores de 45 años en el momento del diagnóstico son una población particularmente vulnerable, por menopausia prematura. También aumenta la enfermedad tromboembólica venosa ${ }^{23}$.

- Arritmias: algunas pacientes con CM metastásico reciben inhibidores CDK 4/6, como el ribociclib, que 


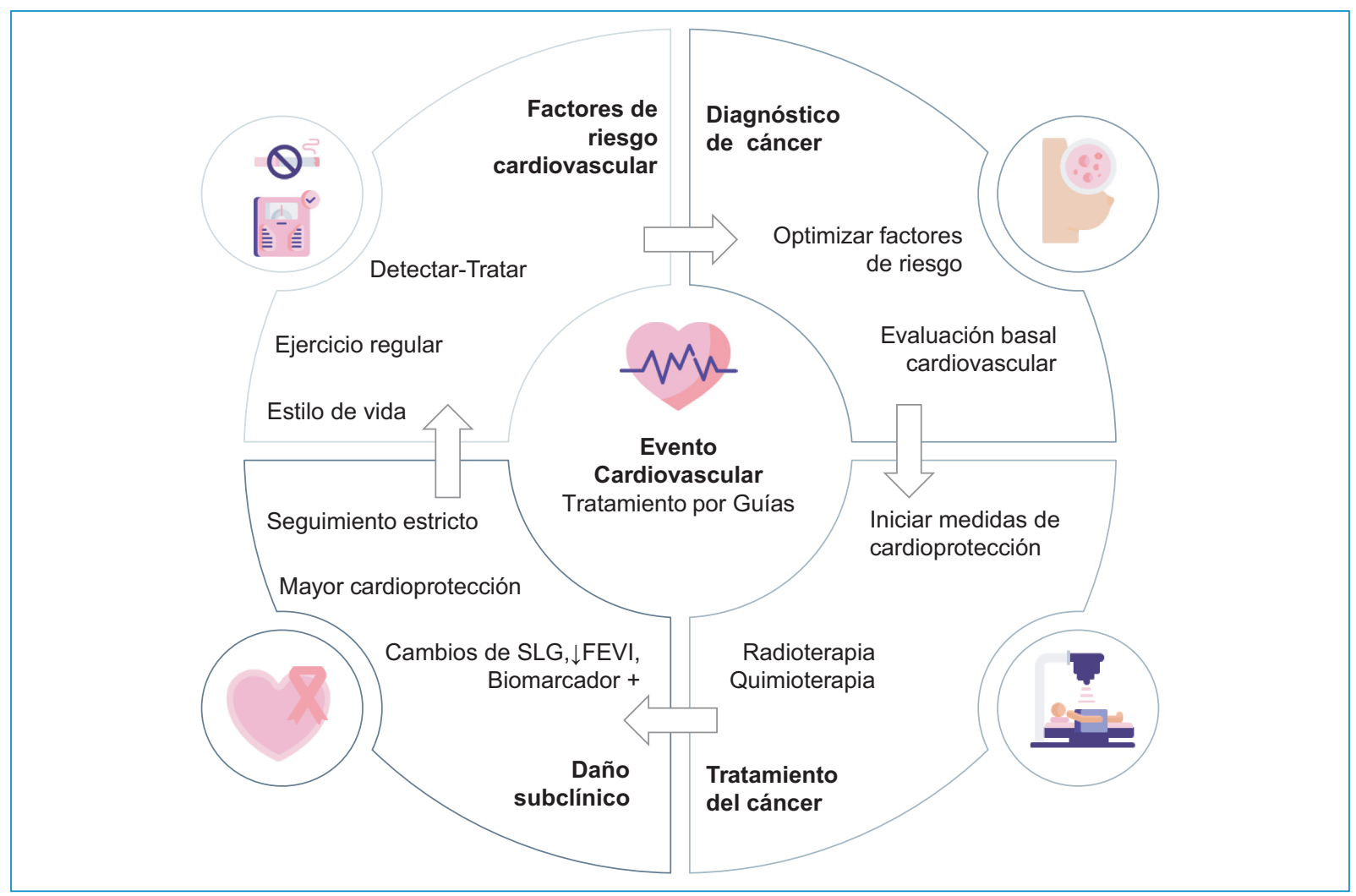

Figura 2. Tratamiento de la enfermedad cardiovascular en cáncer. CV: cardiovascular; DL: dislipidemia; DM: diabetes mellitus; EAC: enfermedad arterial coronaria; EAP: enfermedad arterial periférica; FEVl: fracción de eyección de ventrículo izquierdo; HTA: hipertensión arterial; SLG: srain longitudinal global. Contenido adaptado de: Graffagnino J, et al. ${ }^{38}$

produce un QTc prolongado y se debe monitorizar con electrocardiograma. El intervalo QT y los FR asociados a su prolongación se deben evaluar antes y durante el tratamiento (por ejemplo, desequilibrio hidroelectrolítico, hipotiroidismo, medicamentos, antecedentes familiares, edad avanzada, enfermedad cardiaca, etc.). Un QTc > $460 \mathrm{~ms}$ se sugiere como el límite superior normal en las mujeres, y un QTc > 500 ms con un cambio en el QT > $60 \mathrm{~ms}$ es una señal de alarma porque la torsade de pointes raramente ocurre cuando el QTc es $<500 \mathrm{~ms}$. El QTc se debe medir antes del ingreso, 7-15 días después del o de un cambio de dosis, mensualmente por 3 meses y luego de manera periódica. Si durante el tratamiento se presenta un QTc > $500 \mathrm{~ms}$ u ocurre un cambio en el QT > $60 \mathrm{~m}$, se recomienda suspender temporalmente el medicamento, corregir las anormalidades electrolíticas y controlar otros factores que prolonguen el QTc $^{20}$. Las arritmias por antraciclinas pueden ocurrir antes, durante o después del tratamiento. El manejo debe ser individualizado y las decisiones de uso de antiarrítmicos o de dispositivos se deben realizar teniendo en cuenta la esperanza de vida, la calidad de vida y los riesgos de complicaciones ${ }^{20}$.

\section{Estrategias de tratamiento y seguimiento a largo plazo en pacientes sobrevivientes de cáncer de mama}

Las mujeres tratadas con quimioterapia basada en antraciclinas tienen riesgo durante toda su vida de presentar disfunción del ventrículo izquierdo e insuficiencia cardiaca. El lapso entre el tratamiento y el desarrollo de insuficiencia cardiaca puede ser muy largo ( $>10$ años). Así, las pacientes asintomáticas tratadas con terapia cardiotóxica, particularmente con antraciclinas, pueden presentar disfunción del ventrículo izquierdo e insuficiencia cardiaca. Se recomienda una evaluación periódica con imágenes cardiacas y determinación de biomarcadores, como el péptido natriurético $\mathrm{B}$. Cualquier síntoma sugestivo de insuficiencia cardiaca debe ser investigado, y no se recomienda la descontinuación del tratamiento cardioprotector. Si la paciente presenta 
disfunción ventricular izquierda, el tratamiento es el indicado en las guías de insuficiencia cardiaca ${ }^{20}$.

Tradicionalmente, la toxicidad cardiovascular en los sobrevivientes de cáncer se ha enfocado en especial en la disfunción cardiaca que predispone a la insuficiencia cardiaca. Las consecuencias adversas del tratamiento del cáncer, directas e indirectas, se extienden más allá del corazón para afectar el eje muscular-cardiovascular-esquelético. La función cardiovascular global integrada disminuye durante la exposición a varios protocolos combinados y puede no recuperarse después de la suspensión del tratamiento. Las pacientes con CM de 40 a 50 años tienen un nivel de entrenamiento cardiovascular que es entre un $30 \%$ y un $32 \%$ menor que en mujeres sedentarias saludables de la misma edad. Los estudios aleatorizados que han explorado prescripciones de ejercicio en varias poblaciones con cáncer durante y después de la terapia primaria adyuvante indican que el ejercicio puede atenuar la declinación del entrenamiento cardiovascular. El entrenamiento físico después de terminar la terapia adyuvante aumenta significativamente el entrenamiento cardiovascular comparado con el cuidado usual ${ }^{30}$. En un estudio se reportó que, en 152 sobrevivientes de CM (177 \pm 167 semanas después de la cirugía), el entrenamiento físico aeróbico y de resistencia realizado una vez por semana resultó en una mejoría significativa del entrenamiento cardiovascular $(p<0.001)^{30}$. Colectivamente, las investigaciones actuales indican que los modelos de rehabilitación son realizables y pueden mejorar el entrenamiento cardiovascular, la fuerza muscular y la calidad de vida en las sobrevivientes de CM. También se recomienda un control intensivo de los FR cardiovasculares, así como un estilo de vida saludable.

\section{Conclusiones}

El manejo multimodal oncológico del $\mathrm{CM}$ ha mejorado significativamente el pronóstico y la sobrevida de las pacientes con esta enfermedad, de tal forma que las sobrevivientes del $\mathrm{CM}$ están muriendo más por ECV que por su enfermedad de base. Los resultados a largo plazo de cada paciente pueden mejorarse mediante un trabajo en equipo entre oncología y cardiología. El objetivo en las pacientes con CM debe ser un adecuado seguimiento para facilitar el tratamiento del cáncer y evitar interrupciones precoces e inapropiadas de este, así como detectar, prevenir y manejar las complicaciones cardiovasculares.

Para ello se debe implementar un protocolo que incluya la evaluación antes de la quimioterapia y el cálculo del riesgo cardiovascular, el seguimiento clínico con biomarcadores e imágenes durante la quimioterapia y la radioterapia, realizar la referencia temprana a equipos multidisciplinarios de cardio-oncología, el uso de terapia médica y el seguimiento a largo plazo.

\section{Financiamiento}

Los autores declaran que no recibieron ninguna financiación para la realizar esta publicación.

\section{Conflicto de intereses}

Los autores declaran que no tienen conflictos de interés para escribir este artículo.

\section{Responsabilidades éticas}

Protección de personas y animales. Los autores declaran que para esta investigación no se han realizado experimentos en seres humanos ni en animales.

Confidencialidad de los datos. Los autores declaran que en este artículo no aparecen datos de pacientes.

Derecho a la privacidad y consentimiento informado. Los autores declaran que en este artículo no aparecen datos de pacientes.

\section{Bibliografía}

1. Mehta LS, Watson KE, Barac A, Beckie TM, Bittner V, Cruz-Flores S, et al. American Heart Association Cardiovascular Disease in Women and Special Populations Committee of the Council on Clinical Cardiology; Council on Cardiovascular and Stroke Nursing; and Council on Quality of Care and Outcomes Research. Cardiovascular disease and breast cancer: where these entities intersect: A scientific statement from the American Heart Association. Circulation. 2018;137:e30-e66.

2. Harbeck N, Penault-Llorca F, Cortes J, Gnant M, Houssami N, Poortmans $P$, et al. Breast cancer. Nature Rev Dis Prim. 2019;5:1-31.

3. Bray F, Ferlay J, Soerjomataram I, Siegel R, Torre L, Jemal A. Global cancer statistics 2018: GLOBOCAN estimates of incidence and mortality worldwide for 36 cancers in 185 countries. CA Cancer J Clin. 2018; 68:394-424

4. Valachis A, Nilsson C. Cardiac risk in the treatment of breast cancer: assessment and management. Breast Cancer. 2015;7:21-35.

5. Siegel R, Miller K, Jemal A. Cancer statistics, 2020. CA Cancer J Clin. 2020;70:7-30.

6. Torre L, Islami F, Siegel R, Ward E, Jemal A. Global cancer in women: burden and trends. Cancer Epidemiol Biomarkers Prev. 2017;26:444-57.

7. Hooning MJ, Botma A, Aleman BM, Baaijens MH, Klijn JG, Taylor CW, et al. Long-term risk of cardiovascular disease in 10-year survivors of breast cancer. J Natl Cancer Inst. 2007;99:365-75.

8. Barish R, Lynce F, Unger K, Barac A. Management of cardiovascular disease in women with breast cancer. Circulation. 2019;139:1110-20.

9. Nicolazzi MA, Carnicelli A, Fuorlo M, Scaldaferri A, Masetti R, Landolfi R, et al. Anthracycline and trastuzumab-induced cardiotoxicity in breast cancer. Eur Rev Med Pharmacol Sci. 2018;22:2175-85.

10. Mantarro S, Rossi M, Bonifazi M, D’Amico R, Blandizzi C, La Vecchia C, et al. Risk of severe cardiotoxicity following treatment with trastuzumab: a metaanalysis of randomized and cohort studies of 29,000 women with breast cancer. Intern Emerg Med. 2016;11:123-40.

11. Haddy N, Diallo S, El-Fayed CH, Schwartz B, Pein F, Hawkins M, et al. Diseases following childhood cancer treatment cohort study. Circulation. 2016;136:31-8.

12. López-Fernández T, Martín A, Santabella A, Montero A, García R. Cardio-onco-hematología en la práctica clínica. Documento de consenso y recomendaciones. Rev Esp Cardiol. 2017;70:474-86. 
Rev Colomb Cardiol. 2021;28(3)

13. Goff Jr DC, Lloyd-Jones DM, Bennett G, Coady S, D'Agostino RB, Gibbons $\mathrm{R}$, et al. 2013 ACC/AHA guideline on the assessment of cardiovascular risk: a report of the American College of Cardiology/American Heart Association Task Force on Practice Guidelines. J Am Coll Cardiol. 2014:63:2935e59.

14. Curigliano G, Lenihan D, Fradley M, Ganatra S, Barak A, Blaes A, et al. Management of cardiac disease in cancer patients throughout oncological treatment: ESMO consensus recommendations. Ann Oncol. 2020;31:171-90.

15. Horacek JM, Pudil R, Jebavy L, Tichy M, Zak P, Maly J. Assessment o anthracycline induced cardiotoxicity with biochemical markers. Exp Oncol. 2007;29:309-13.

16. Navarrete S. Cáncer y cardiotoxicidad en la mujer. Rev Colomb Cardiol. 2018;25(Supl 1):144-53.

17. Plana JC, Galderisi M, Barac A, Ewer MS, Ky B, Scherrer-Crosbie M, et al. Expert consensus for multimodality imaging evaluation of adult patients during and after cancer therapy: a report from the American Society of Echocardiography and the European Association of Cardiovascular Imaging. J Am Soc Echocardiogr. 2014;27:911-39.

18. Patnaik JL, Byers T, DiGuiseppi C, Dabelea D, Denberg TD. Cardiovascular disease competes with breast cancer as the leading cause of death for older females diagnosed with breast cancer: a retrospective cohort study. Breast Cancer Res. 2011;13:R64.

19. Gilchrist SC, Barac A, Ades PA, Alfano CM, Franklin BA, Jones LW, et al. Cardio-oncology rehabilitation to manage cardiovascular outcomes in cancer patients and survivors. A scientific statement from the American Heart Association. Circulation. 2019;139:e997-e1012.

20. Zamorano JL, Lancelotti P, Rodríguez D, Aboyans V, Asteggiano R, Galderisi M, et al. 2016 ESC position paper on cancer treatments and cardiovascular toxicity developed under the auspices of the ESC Committee for Practice Guidelines. Eur Heart J. 2016;37:2768-801.

21. Bhave M, Shah AN, Akhter N, Rosen ST. An update on the risk prediction and prevention of anticancer therapy-induced cardiotoxicity. Curr Opin Oncol. 2014;26:590-9.

22. Kalam K, Marwick TH. Role of cardioprotective therapy for prevention of cardiotoxicity with chemotherapy: a systematic review and meta-analysis. Eur J Cancer. 2013;49:2900-9.

23. van Dalen EC, Caron HN, Dickinson HO, Kremer LC. Cardioprotective interventions for cancer patients receiving anthracyclines. Cochrane $\mathrm{Da}$ tabase Syst Rev. 2011;(2):CD003917.

24. ZINECARD® (dexrazoxane for injection). New York, NY: Pfizer; 2012. (Consultado 16 abril 2020.) Disponible en: https://www.accessdata.fda. gov/drugsatfda_docs/label/2012/020212s013lbl.pdf.

25. European Medicines Agency recommends restricting the use of dexrazoxane containing medicines. London, United Kingdom: European Medicines Agency; June 23, 2011. (Consultado 16 abril 2020.) Disponible en: http://www.ema.europa.eu/docs/en_GB/document_library/Press_release/2011/06/WC500108013. pdf.

26. van Dalen EC, van der Pal HJ, Caron HN, Kremer LC. Different dosage schedules for reducing cardiotoxicity in cancer patients receiving anthracycline chemotherapy. Cochrane Database Syst Rev. 2009;(4):CD005008.
27. Smith LA, Cornelius VR, Plummer CJ, Levitt G, Verrill M, Canney P, et al. Cardiotoxicity of anthracycline agents for the treatment of cancer: systematic review and meta-analysis of randomised controlled trials. BMC Cancer. 2010;10:337.

28. Kalay N, Basar E, Ozdogru I, Er O, Cetinkaya Y, Ali Dogan, et al. Protective effects of carvedilol against anthracycline-induced cardiomyopathy. J Am Coll Cardiol. 2006;48(11):2258-2262

29. Kaya MG, Ozkan M, Gunebakmaz O, Akkaya H, Kaya EG, Akpek M, et al. Protective effects of nebivolol against anthracycline-induced cardiomyopathy: a randomized control study. Int J Cardiol 2013; 167(5):2306-2310

30. Seicean S, Seicean A, Alan N, Plana JC, Budd GT, Marwick TH. Cardioprotective effect of $\beta$-adrenoceptor blockade in patients with breast cancer undergoing chemotherapy: follow-up study of heart failure. Circ Heart Fail. 2013 May; 6(3):420-6

31. Munoz J, Sheqwara J, Arango B, Ali HY, Weaver DW. The role of beta-blockers and ACE inhibitors in the prevention of trastuzumab-related cardiotoxicity. J Clin Onc. 2010;28(15_suppl):555-555.

32. Gulati G, Heck SL, Ree AH, Hoffmann P, Schulz-Menger J, Fagerland $\mathrm{MW}$, et al. Prevention of cardiac dysfunction during adjuvant breast cancer therapy (PRADA): a $2 \times 2$ factorial, randomized, placebo-controlled, double-blind clinical trial of candesartan and metoprolol. Eur Heart J. 2016;37:1671-80. doi: 10.1093/eurheartj/ehw022

33. Pituskin E, Mackey JR, Koshman S, Jassal D, Pitz M, Haykowsky MJ, et al. Multidisciplinary Approach to Novel Therapies in Cardio-Oncology Research (MANTICORE 101-Breast): A Randomized Trial for the Prevention of Trastuzumab-Associated Cardiotoxicity. J Clin Oncol. 2017:35:870-7. doi: 10.1200/JCO.2016.68.7830

34. Guglin M, Krischer J, Tamura R, Fink A, Bello-Matricaria L, McCaskiII-Stevens W, et al. Randomized Trial of Lisinopril Versus Carvedilol to Prevent Trastuzumab Cardiotoxicity in Patients with Breast Cancer. J Am Coll. Cardiol. 2019;73:2859-68.

35. Blanter JB, Frishman WH. The Preventive Role of Angiotensin Converting Enzyme Inhibitors/Angiotensin-II Receptor Blockers and $\beta$-Adrenergic Blockers in Anthracycline- and Trastuzumab-Induced Cardiotoxicity. Cardiol Rev. 2019;27(5):256-259.

36. Akpek M, Ozdogru I, Sahin O, Inanc M, Dogan A, Yazici C, et al. Protective effects of spironolactone against anthracycline-induced cardiomyopathy: effects of spironolactone on anthracycline cardiomyopathy. Eur J Heart Fail. 2015;17(1):81-9.

37. Brown SA, Okwuosa TM, Barac A, Volgman AS. The role of angiotensin-converting enzyme inhibitors and beta blockers in primary prevention of cardiac dysfunction in breast cancer patients. J Am Heart Assoc. 2020;9:e015327.

38. Graffagnino J, Kondapalli L, Arora G, Hawi R, Lenneman CG. Strategies to prevent cardiotoxicity. Curr Treat Options Oncol. 2020;21:32.

39. Wolin KY, Schwartz AL, Matthews CE, Courneya KS, Schmitz KH. Implementing the exercise guidelines for cancer survivors. J Support Oncol. 2012;10(5):171-7. 